\title{
Investigation of Aging in the HERA-B Outer Tracker Drift Tubes
}

\author{
H. Kolanoski \\ representing the HERA-B Outer Tracker Group \\ Humboldt University Berlin
}

\begin{abstract}
The HERA-B Outer Tracker consists of drift tubes folded from polycarbonate foil and is operated with $\mathrm{Ar}^{\mathrm{CF}_{4}} \mathrm{CO}_{2}$ as drift gas. The detector has to stand radiation levels which are similar to LHC conditions. The first prototypes exposed to radiation in HERA-B suffered severe radiation damage due to the development of self-sustaining currents (Malter effect). In a subsequent extended R\&D program major changes to the original concept for the drift tubes (surface conductivity, drift gas, production materials) have been developed and validated for use in harsh radiation environments. In the test program various aging effects - like Malter currents, gain loss due to anode aging and etching of the anode gold surface - have been observed and cures by tuning of operation parameters have been developed.
\end{abstract}

\section{INTRODUCTION}

HERA-B is a fixed target experiment for studying CP violation in B-meson systems using an internal wire target in the proton beam of HERA [1]. To reach the necessary production rate of $b$ quarks an average of 4 interactions per bunch at a frequency of about $10 \mathrm{MHz}$ ( $96 \mathrm{~ns}$ bunch separation) has to be generated. This leads to a high particle density with a radial distribution of the particle flux ( $R$ is the distance from the beam) as shown in Fig. 1:

$$
\phi \approx \frac{2 \cdot 10^{7}}{\mathrm{~cm}^{2} \mathrm{~s}} \frac{1}{R^{2} / \mathrm{cm}^{2}}
$$

The main detector components are a silicon vertex detector, a magnet $(2.2 \mathrm{Tm})$, a main tracker with MSGCs in the inner and drift tubes in the outer part, 'High- $\mathrm{P}_{T}$ ' trigger chambers, a RICH, a TRD, an electro-magnetic calorimeter and a muon detector with drift tubes. The detector covers a forward angular range of $220 \mathrm{mrad}$ in the bending plane of the magnet and 160 mrad vertically.

The high irradiation level in HERA-B led to several, partly quite painful experiences with aging problems in the gaseous detectors. We believe that these problems have been solved, either by changing the materials and technologies (e. g. introducing GEM foils in the MSGC) or by going to a different detector type (e.g. the photon detection of the RICH is now done by photomultipliers rather than the proposed gaseous photon detector).

In the following we report on the different aging problems which HERA-B had encountered in the Outer Tracker drift chambers and how these problems have been solved.

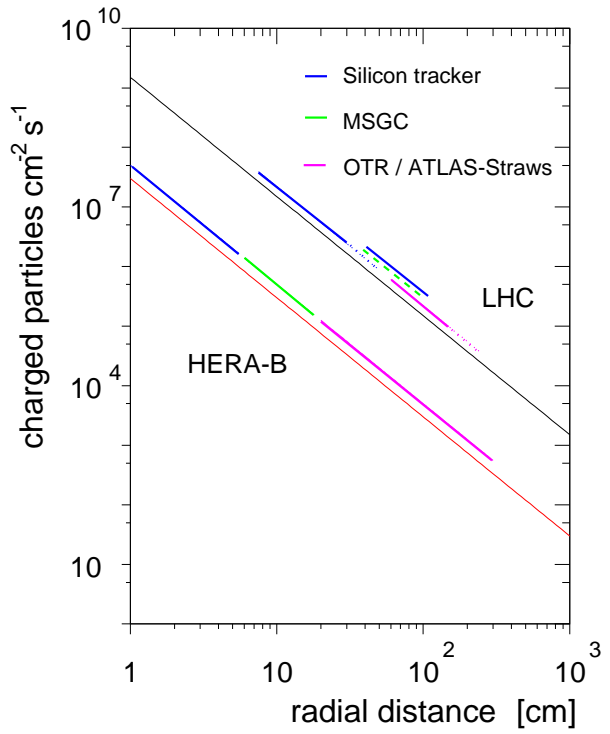

Figure 1: Particle flux as a function of the radial distance from the beam for HERA-B and LHC experiments. The flux in the Outer Tracker region is comparable to the flux which the ATLAS TRT straws will see in LHC.

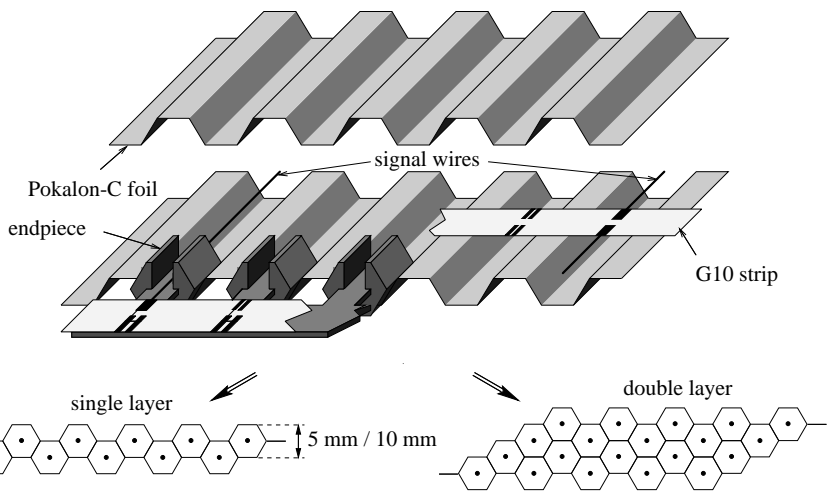

Figure 2: Illustration of the honeycomb technology: The folded cathode foil is placed into a template, the wires are strung and soldered unto pads on G10 strips, another folded foil is then glued to the layer below such that both form hexagonal cells. In the lower part of the figure the cross sections of single and double layer modules is shown.

\section{THE HERA-B OUTER TRACKER}

The Outer Tracker of HERA-B [2] consists of 13 planar superlayers of drift tube modules comprising a total of about 115000 channels. The detector acceptance starts at a radial distance of $19 \mathrm{~cm}$ to the beam. Together with the Inner Tracker it allows for a precise momentum measurement and provides fast track recognition on the first trigger level. Pattern recognition in a dense particle environment and a precise momentum measurement require a position resolution of about 
$200 \mu \mathrm{m}$. The first level trigger requires a high hit efficiency and a fast signal collection within the bunch separation of 96 ns. The choice of the materials and the chamber technology were driven by the requirements of radiation hardness, minimal radiation length and mass producibility.

The drift tubes are folded from $75 \mu \mathrm{m}$ thick carbon-loaded polycarbonate foil (Pokalon-C) with an hexagonal cross section (honeycomb tubes, see Fig. 2) serving as cathode. As a result of the R\&D work described below the foil was coated by a thin $\mathrm{Au} / \mathrm{Cu}$ layer (Au: $40 \mathrm{~nm}, \mathrm{Cu}: 50 \mathrm{~nm}$ ). The anode wire is made of gold-plated tungsten and has a diameter of $25 \mu \mathrm{m}$.

The inner diameter of the cells is $5 \mathrm{~mm}$ in the most exposed sections and $10 \mathrm{~mm}$ further outside such that the occupancies do not exceed about $20 \%$. Nearly all aging tests described below have been done with $5 \mathrm{~mm}$ cells which turned out to be more susceptible to aging effects. The detector is built of modules with a length of up to $4.5 \mathrm{~m}$. Longitudinally the anode wires are supported at least every $60 \mathrm{~cm}$ by 'G10 strips' (the material is FR4) as shown in Fig. 2.

The chambers have to stand a very high particle flux (see Fig. 1) which is in the hottest area about $2 \cdot 10^{5} \mathrm{~cm}^{-2} \mathrm{~s}^{-1}$. The drift tubes are longitudinally subdivided to limit the single channel occupancy to about 20\% (the shortest segmentation near the beam is $20 \mathrm{~cm}$ ). The charge collected on the anode wires will be up to $0.6 \mathrm{C} / \mathrm{cm}$ per year. Because of the planar detector geometry the front-end amplifiers (ASD-8) can be placed away from the beam pipe at a radiation load below about $50 \mathrm{~Gy} /$ year.

Since the first level trigger requires that the drift signals be collected within the bunch separation time of $96 \mathrm{~ns}$, a fast drift gas containing $\mathrm{CF}_{4}$ had to be chosen. As a result of the R\&D studies the final choice is $\mathrm{Ar} \mathrm{CF}_{4} \mathrm{CO}_{2}$ (65:30:5). Operating at a gain of about $3 \cdot 10^{4}$ the average drift velocity is about 80 $\mu \mathrm{m} / \mathrm{ns}$ which allows the track signals to be collected between two bunch crossings.

A brief summary of materials used for the final construction

Table 1

Some materials used for the construction and important operation parameters of the Outer Tracker.

\begin{tabular}{|l|l|}
\hline component & material \\
\hline \hline cathode & $\mathrm{Au} / \mathrm{Cu}(40 / 50 \mathrm{~nm})$ coated Pokalon-C \\
\hline anode & $\begin{array}{l}25 \mu \mathrm{m} \text { Au coated tungsten wire } \\
(6 \% \text { gold in weight })\end{array}$ \\
\hline support strips & FR4, Sn coated solder pads \\
\hline glue & STYCAST type 1266 / CATALYST 9 \\
\hline endpieces & Noryl \\
\hline
\end{tabular}

\begin{tabular}{|l|l|}
\hline parameter & value \\
\hline \hline drift gas & $\mathrm{Ar} / \mathrm{CF}_{4} / \mathrm{CO}_{2}(65 / 30 / 5)$ \\
\hline gain & $2.5 \times 10^{4}($ at $1700 \mathrm{~V})$ \\
\hline max. particle flux & $10^{5} \mathrm{~cm}^{-2} \mathrm{~s}^{-1}$ \\
\hline max. charge/year & $0.6 \mathrm{C} / \mathrm{cm}$ \\
\hline gas flow & 1 box volume per hour \\
\hline
\end{tabular}

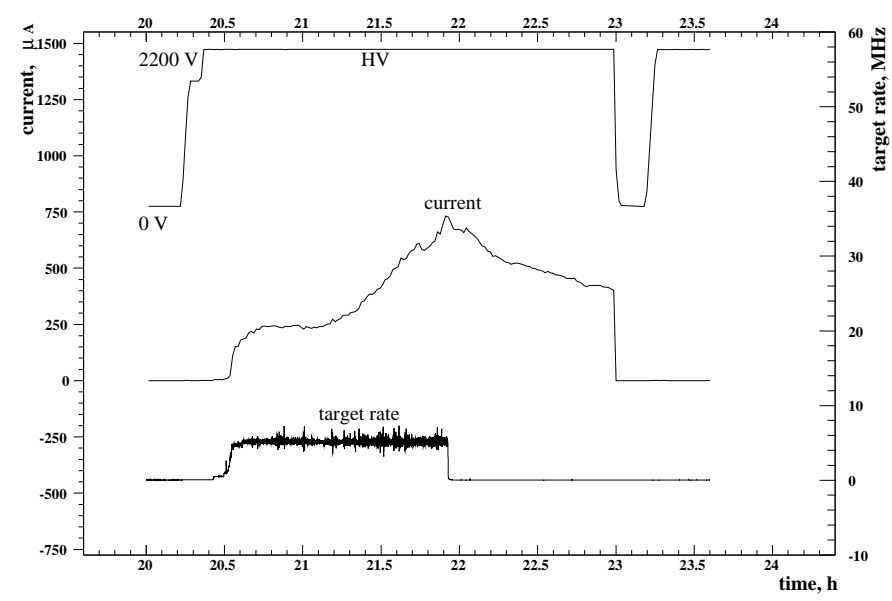

Figure 3: Example for the development of a Malter current in prototype chambers in HERA-B (1997). The cathode foil was uncoated Pokalon-C and the drift gas $\mathrm{Ar} \mathrm{CF}_{4} \mathrm{CH}_{4}$. The plot shows the high voltage on the chamber, the current and the target rate which is proportional to the radiation load on the chamber.

of the Outer Tracker modules and of the parameters which are most important for the operation of the detector is given in Table 1.

\section{Aging Studies For the Honeycomb DRIFT TUBES}

\section{A. Malter Currents in Prototype tests in HERA-B}

The first prototype honeycomb chambers were made of uncoated Pokalon-C foil and have been tested in drift gases containing methane, initially $\mathrm{CF}_{4} \mathrm{CH}_{4} \quad(80: 20)$ and later $\mathrm{Ar} \mathrm{CF}_{4} \mathrm{CH}_{4}$ (74:20:6).

Small prototypes with a cell length of $10 \mathrm{~cm}$ have been tested with $\mathrm{X}$-rays from a tube with a molybdenum anode $(35 \mathrm{kV})$. Up to a collected charge of $4.5 \mathrm{C} / \mathrm{cm}$ in the honeycomb cells no aging effects in form of gain losses or visible deposits on the electrodes have been observed.

The more surprising it was that chambers which have been installed in HERA-B started drawing excessive currents after only hours of operation under standard working conditions. The corresponding charge collected on the anode was only a few $\mathrm{mC} / \mathrm{cm}$. Figure 3 shows a typical example of these currents: At some point in time irradiated chambers start drawing increasing currents which usually develop within hours to a level which goes beyond the current limitation of the HV system. These currents are persistent when one switches off the radition, i. e. in HERA-B the target rate, and vanish only after turning off the high voltage. Once the effect has been observed it is easily ignited again by irradiation, even with X-rays.

Such a behaviour of the currents is reminiscent of the Malter effect: An insulating layer on the cathode inhibits the neutralisation of the positive ions arriving from the avalanche at the anode, a charge layer accumulates and generates a strong electric field across the insulating layer. At some point the field becomes strong enough to extract electrons out of the cathode with enough energy to pass the charge layer and to reach the 
anode. Here they generate an avalanche and thus contribute to a self-sustaining current.

We investigated if the Malter effect could explain our observations. An optical inspection of the cathode surface revealed changes indicating deposits on the foil. An analysis employing the ESCA method (electron emission spectroscopy for chemical analysis) showed $\mathrm{CN}, \mathrm{CF}$ and $\mathrm{CO}$ components in a polymerized form. While on non-irradiated foils the undisturbed $\mathrm{CO}$ groups from the polycarbonat (Bisphenol $\mathrm{A}$ ) were dominating in the ESCA spectrum, on irradiated foils the peaks showed a broadening which is characteristic for plasma polymers. The occurrence of nitrogen in the spectra of used Pokalon-C could be an indication for out-gassing of the epoxy used for glueing the foil (Araldite, the hardener contains $\mathrm{CN}$ components).

Searching for a remedy of this problem we investigated various treatments of the cathode surface: plasma etching of the foil and coating with graphite and various metals $(\mathrm{Au}, \mathrm{Cu}$, $\mathrm{Cr}$ ). In all cases Malter currents could be avoided. This is not easily explainable by the influence of differing conductivities since for the soot-loaded Pokalon-C the resistivity of an area was measured using metal contacts to be about $120 \Omega / \square$. This value is not substancially modified by an additional graphite coating.

To find out what gave rise to the build-up of a polymerisation layer we inspected more closely the surface of the foil. Using scanning electron microscopy (SEM) we found that for low electron energies the foil behaved like an insulating layer while at a transition energy of about $0.9 \mathrm{keV}$ the foil surface looked conductive. The effect was only seen for untreated Pokalon-C foils. This observation could be interpreted such that the foil has a thin insulating layer of less than about $100 \mathrm{~nm}$ which may be a result of the production procedure.

Another puzzle was the fact that the Malter currents have not been observed in the aging tests under X-ray irradiation. This could have been partially due to the small size of the detector and/or the size of the irradiated area or other specialties of the test setup. However, the most pronounced effect which could clearly be established is a strong dependence on the nature of the beam. Searching for a suitable test beam we found that in addition to X-rays electrons $(2.5 \mathrm{MeV})$, low energy protons (about $20 \mathrm{MeV}$ ) and low energy $\alpha$ 's (about $6 \mathrm{MeV}$ (source) and $28 \mathrm{MeV}$ (beam)) could not or not clearly generate the Malter effect. However, the effect observed in HERA-B could be reproduced in a $350 \mathrm{MeV}$ pion/proton beam (PSI) and in a $100 \mathrm{MeV} \alpha$ beam (Karlsruhe). The ionisation density of the beam particles cannot be an explanation for the beam dependence since the low energy protons and, even more so, $\alpha$ 's have a much higher ionisation density than, for example, the $350 \mathrm{MeV}$ pions. We suspect that the Malter effect is ignited by heavy fragments from nuclear interactions which can have very high ionisation densities, leading to sufficiently strong accumulation of ions on small cathode areas. For example in nuclear interactions with gold the ionisation could be up to about $10^{4}$ times higher than for minimum ionising particles [3]. Indeed the differences of the nuclear cross sections of the particle beams used in these tests appear to support this hypothesis.

As a result of these investigations HERA-B opted for a $\mathrm{Au} / \mathrm{Cu}$ coating $(40 / 50 \mathrm{~nm})$. The copper is applied as a primer layer for better adhesion, gold was chosen because of its known inert behaviour in gaseous detectors. Graphite coating was experimentally not excluded, in fact, we have been running a half-superlayer in the magnet with graphite coated cathodes for more than a year without any problems. Metallic coating appeared to be preferable from the technological point of view (better adhesion). Coating with copper (as the cathode surface) was excluded because of bad experience reported for such cathodes in ${\mathrm{a} \mathrm{CF}_{4}}_{4}$ environment [4].

\section{B. Anode Aging}

After the first observation of Malter currents in the Outer Tracker the drift gas was changed from $\mathrm{CF}_{4} \mathrm{CH}_{4}$ (80:20) to $\mathrm{Ar} \mathrm{CF}_{4} \mathrm{CH}_{4}$ (74:20:6). Amongst other reasons, this choice was driven by the lower $\mathrm{CF}_{4}$ content which makes the gas much cheaper while the requirement on the drift velocity is still fulfilled. With a drift velocity of about $100 \mu \mathrm{m} / \mathrm{ns}$ the gas is fast enough to meet the HERA-B requirements. However, in all tests with this drift gas a fast gain loss after only a few $\mathrm{mC} / \mathrm{cm}$ collected charge on the anodes was observed.

The inspection of the affected chambers showed deposits on the anode wire presumably due to plasma polymerisation of hydrocarbons.

Such fast aging was not observed in $\mathrm{Ar}^{\mathrm{CF}_{4}} \mathrm{CO}_{2}$ (65:30:5) or in $\mathrm{ArCO}_{2}$ (80:20). For the operation in HERA-B $\mathrm{Ar} \mathrm{CO}_{2}$ could not be chosen because it is too slow. The gases containing $\mathrm{CO}_{2}$ were even able to reverse (at least to a certain degree) both the anode and cathode aging effects.

\section{Definition and Validation of the Basic Chamber Parameters}

As a result of the above described investigations new basic parameters for the construction and operation of the Outer Tracker were defined. The three most important changes were:

- cathode: Au coating of the Pokalon-C foil,

- drift gas: $\mathrm{Ar} \mathrm{CF}_{4} \mathrm{CO}_{2}$ (65:30:5), no methane,

- glue: change from Araldite to Stycast.

Concerning the glue we have profitted from out-gassing tests done at CERN [5]. In general care was taken to choose materials which show no out-gassing.

The materials, the construction principle and the operation of chambers has been validated in a $100 \mathrm{MeV} \alpha$ beam at a cyclotron of the Karlsruhe Research Center. This beam has been found to reproduce the Malter effect in chambers with uncoated Pokalon-C cathodes. In the course of the experiment various parameters have been tested:

- cathode surface: uncoated and coated Pokalon-C, straw tubes (ATLAS-type), 


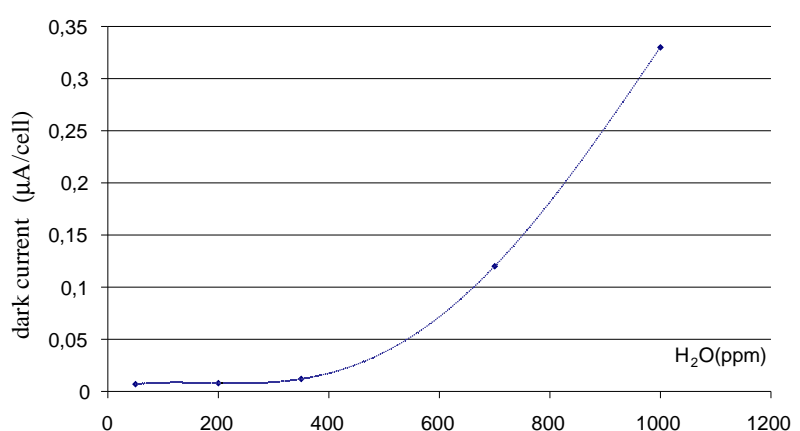

Figure 4: Dark current as a function of the water concentration in the drift gas for an irradiated chamber with G10 strips. At the beginning of the irradiation the current is increasing but after a collected charge of about $0.3 \mathrm{C} / \mathrm{cm}$ the current saturates at a value depending on the water concentration.

- gas mixtures: with and without methane,

- gas flow: tests with $0.1,1,10$ volume exchanges per hour,

- water concentration: about 500 to 3000 ppm of water.

At a collected charge of nearly $1 \mathrm{C} / \mathrm{cm}$ the tested chambers did not show any of the previously encountered aging problems, neither Malter currents nor fast anode aging. However, two new effects were observed which were both connected to the water concentration in the drift gas: strip conductivity and corrosion of the anodes.

\section{Strip Conductivity}

In an initial tests series in Karlsruhe the chambers started to draw currents with ohmic behaviour after being irradiated with a dose of about $300 \mathrm{mC} / \mathrm{cm}$. These currents were caused by the support strips ('G10 strips') which became conductive during irradiation. Inspection of the strips showed strong corrosion both of the epoxy of the strip (the glas fiber texture became quite pronounced) and of the solder points. In these tests the water content in the gas boxes was not well controlled and exceeded $3000 \mathrm{ppm}$ because the windows of the gas box were made of Kapton which is rather transparent for water. After improving the windows the water content could be reduced to a low level. For water concentrations below $500 \mathrm{ppm}$ no dangerous ohmic rest currents were observed (see Fig. 4).

\section{E. Plasma Corrosion of Anodes}

The final tests in the $\alpha$ beam in Karlsruhe were done with a low water concentration in the drift gas. The total exposure was about $0.9 \mathrm{C} / \mathrm{cm}$. Neither Malter currents nor fast anode aging effects were observed for the standard chamber setups. However, the gain as measured with a ${ }^{55} \mathrm{Fe}$ source had dropped in some chambers up to about $10 \%$. After opening such chambers and inspecting the wires it was found that the anode wires have become slightly thicker consistent with causing the observed gain decrease. At some places the gold was peeled off.

In laboratory tests with controlled water concentration this effect could be reproduced: At very low water concentration, below about $50 \mathrm{ppm}$, under a high irradiation level, typically about $0.6 \mu \mathrm{A} / \mathrm{cm}$, and with a relatively low gas flow the anode wires exhibited drastic changes of their gold surface after $\mathrm{O}(100 \mathrm{mC} / \mathrm{cm})$. With increasing accumulated charge the wire first became thicker, then the gold started to peel off exposing to the tungsten which was then readily etched away. Finally, the wires ruptured near the center of the irradiation zone. In this zone the cathode was found to be relatively clean while deposits, mainly containing tungsten, were observed at the border of the irradiated area (diameter about $10 \mathrm{~cm}$ ). In the rupture zone the wires looked badly corroded with only tungsten observable (see Fig. 5)

With a water concentration above about $200 \mathrm{ppm}$ these damages could be completely avoided. An interpretation could be that water neutralizes fluorine radicals such as $\mathrm{F}, \mathrm{CF}_{2}$, $\mathrm{CF}_{3}$ which are generated in the avalanche. The experimental conditions under which this plasma corrosion process occurs have been tested at a limited number of points in the parameter space. The observed dependences are:

- Dry gas: the effect was mainly observed for 20-50 ppm of water (note that the measurement of such a low water concentration is difficult), above $400 \mathrm{ppm}$ the effect was not observed.

- Radiation intensity: the tests have been done at about one order of magnitude higher irradiation levels than expected in HERA-B, mainly around 0.7 and $0.4 \mu \mathrm{A} / \mathrm{cm}$. The results suggest that the damage depends mainly on the accumulated charge rather than the intensity.

- Gas flow: the tests were mainly done at a gas flow of about 1 volume exchange per hour. There were indications that the corrosion effects appear faster for a lower flow. This statement is not very firm because there was a correlation between the gas flow and the water content at very low water concentrations.

These measurements have been done with an open gas system. In another setup a closed gas system was tested together with the purification system as used for the Outer Tracker. Due to the purifiers the water concentration was in this case below $20 \mathrm{ppm}$. Despite this very low water content, no wire rupture was observed up to a charge of $2.35 \mathrm{C} / \mathrm{cm}$ collected at an intensity of about $0.2 \mu \mathrm{A} / \mathrm{cm}$. It is still under study why the two setups show such a different aging behaviour.

Anode etching has also been observed in tests of straws for the ATLAS TRT [6]. However, the dependence on the water concentration seems to be opposite [7].

Independent of these still open questions we could conclude that the detector can be safely run with a water concentration in the drift gas around 200 to $400 \mathrm{ppm}$.

\section{SUMMARY AND CONCLUSION}

The honeycomb drift tubes of the HERA-B Outer Tracker are a new detector type which has been developed (starting only 

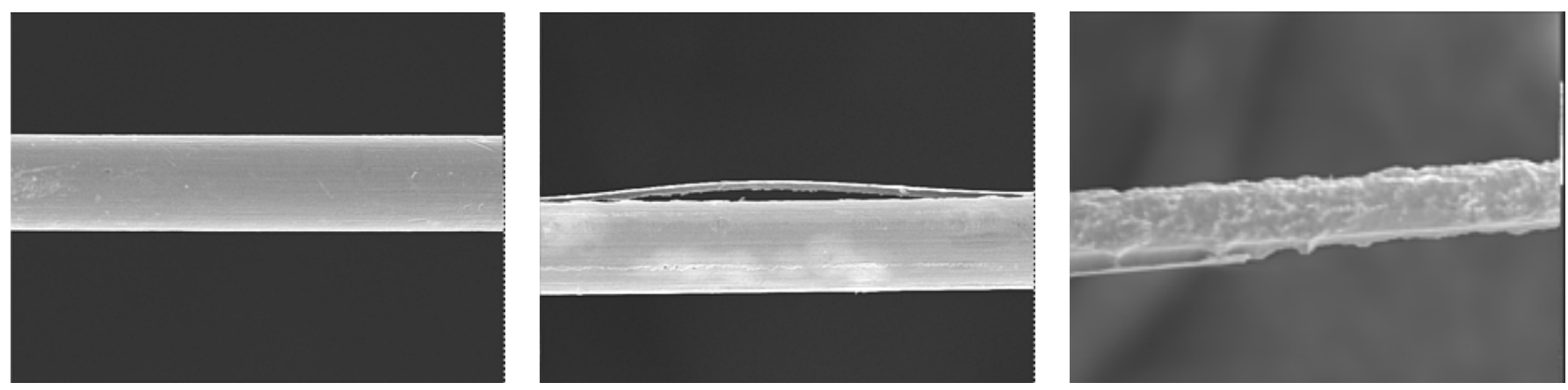

Figure 5: Anode wires samples demonstrating the effect of plasma corrosion: new wire (left), irradiated wire $\left(\mathrm{about} 1 \mathrm{C} / \mathrm{cm}, \mathrm{c}\left(\mathrm{H}_{2} \mathrm{O}\right) \approx 50 \mathrm{ppm}\right)$ where the gold coating starts to peel off (middle), completely corroded wire which ruptured close to where the picture was taken (right).

Table 2

Radiation induced aging problems observed in tests and the solutions found for the Outer Tracker.

\begin{tabular}{|l|l|l|}
\hline Effect & Condition & Solution \\
\hline \hline Malter effect & uncoated foil, $\mathrm{Ar} \mathrm{CF}_{4} \mathrm{CH}_{4}$ & $\mathrm{Au}$ coating, $\mathrm{CH}_{4} \rightarrow \mathrm{CO}_{2}$ \\
\hline anode aging & $\mathrm{Ar} \mathrm{CF}_{4} \mathrm{CH}_{4}$ & $\mathrm{CH}_{4} \rightarrow \mathrm{CO}_{2}$, no out-gassing materials \\
\hline strip conductivity & $\mathrm{H}_{2} \mathrm{O}>500 \mathrm{ppm}$ & $\mathrm{H}_{2} \mathrm{O}$ control \\
\hline anode corrosion & $\mathrm{H}_{2} \mathrm{O}<100 \mathrm{ppm}$ & $\mathrm{H}_{2} \mathrm{O}$ control \\
\hline
\end{tabular}

in 1995!) to meet the requirements of the HERA-B experiment. Like the other gaseous detectors in the experiment the Outer Tracker encountered many very severe aging problems due to the high irradiation level in the HERA-B detector. These problems have been solved by now (Table 2) and the Outer Tracker is close to reaching the design goals. The honeycomb cell structure has proven to be suited for mass production and to be a good solution for running in the HERA-B environment (for the latest status see [8]).

The main lessons learnt from the extensive R\&D for the radiation hardness of the detector can be summarized as follows:

- Radiation damages depend on the particle type and energy, nuclear interactions in hadronic beams are in general dangerous.

- Surface properties of the cathode can have an important influence which are not sufficiently described by a macroscopically measured surface conductivity.

- Methane (and possibly other hydrocarbons) in $\mathrm{CF}_{4}$ gas mixtures is very dangerous and should be avoided. No anode aging from polymerisation was observed when $\mathrm{CH}_{4}$ was replaced by $\mathrm{CO}_{2}$.

- The water concentration in gases containing $\mathrm{CF}_{4}$ is a crucial parameter, probably it neutralizes aggressive radicals. On the other hand, too much water can adversely affect the operation by introducing conductive bridges etc.

Some of these lessons have become common wisdom. For example, the beam dependence of radiation damages has become a standard consideration for the LHC detector development. Thus the partly quite painful experience of HERA-B with radiation damages may at the end help other experiments to avoid such problems.

\section{REFERENCES}

[1] E. Hartouni et al., HERA-B Design Report, DESY-PRC 95/01 (1995).

[2] M. Capeáns, 'Status of the Outer Tracker for the HERA-B Experiment', Nucl. Instrum. Meth. A446 (2000) 317.

[3] G. Bayatian et al., 'CMS - The Tracker Project, Technical Design Report', appendix A, CERN/LHCC 98-6, 1998.

[4] K. M.Ecklund et al., 'Etching of Copper Coated Mylar Tubes with $\mathrm{CF}_{4}$ Gas', SLAC-PUB-7175G, SLAC-PUB7175, May 1996.

[5] R. Bouclier et al., 'Effects of outgassing from some materials on gas chamber aging', Nucl. Instrum. Meth. A350 (1994) 464;

A. Barr et al., 'Construction, test and operation in a high intensity beam of a small system of micro-strip gas chambers', Nucl. Instrum. Meth. A403 (1998) 31.

[6] T. Ferguson et al., 'Possible New Mechanism for Anode Wire Aging in Gas-Filled Detectors', PNPI preprint EP-461999 2331, Gatchina 1999.

[7] A. Romaniouk, private communication.

[8] HERA-B Collaboration, 'HERA-B - Report on Status and Prospects', DESY-PRC 00/04, October 2000. 